\title{
ANUARIO DE RATIOS FINANCIEROS SECTORIALES EN MÉXICO PARA ANÁLISIS COMPARATIVO EMPRESARIAL
}

\section{YEARBOOK SECTORAL FINANCIAL RATIOS IN MEXICO FOR BUSINESS BENCHMARKING}

\section{Deyanira Bernal-Domínguez1 y Oriol Amat-Salas2}

Doctora en Ciencias Sociales por la UAS1. Dr. En Ciencias Económicas por la UAB International

\section{RESUMEN}

El análisis financiero a través de ratios es una herramienta útil para mejorar el desempeño de las organizaciones. Las bases de datos de información financiera en México son limitadas de ahí la propuesta de una publicación anual de ratios financieros por empresa y promedio sectorial. Los objetivos de este trabajo de investigación son: describir los ratios financieros ideales con mayor potencial predictivo y sus fórmulas, así como el diseño de un instrumento de investigación para medir la pertinencia de la publicación mencionada. Se aplicó la metodología descriptiva, por lo que, se seleccionaron con base en el análisis de estudios empíricos, los diversos ratios ideales de liquidez, endeudamiento, gestión de activos, ciclo económico, rendimiento y capacidad de autofinanciación. El cuestionario que se presenta contiene 43 reactivos que será aplicado a una muestra representativa estadísticamente de 46 empresarios en Culiacán, Sinaloa, México.

Palabras clave: base de datos, análisis financiero, indicadores financieros, instrumento de investigación.

\section{SUMMARY}

Financial analysis through ratios is a useful tool for improving organizational performance. Databases of financial

Recibido: 04 de diciembre de 2011. Aceptado: 02 de marzo de 2012. Publicado como ARTíCULO CIENTÍFICO en Ra Ximhai 8 (3): $267-281$ information in Mexico are limited, therefore the importance of an annual publication of financial ratios per company and industry average. The objectives of this research are: describe the financial ratios with higher predictive potential and their formulas, as well as the design of a research instrument for measuring the relevance of the publication. A descriptive methodology was applied selecting through the analysis of empirical studies, several ratios of liquidity, leverage, asset management, business cycle, performance and self-financing. The questionnaire contains 43 reagents to be applied to a statistically representative sample of 46 entrepreneurs in Culiacan, Sinaloa, Mexico.

Keywords: database, financial analysis, financial ratios, research tool.

\section{INTRODUCCIÓN}

Los directivos y cualquier persona interesada en una empresa necesitan conocer la situación en que se encuentra. Para ello, deben analizar continuamente la marcha de la empresa. Una de las partes del análisis integral es la que se puede confeccionar a partir de los estados financieros. Estos informan de la situación y evolución económica y financiera de la empresa. El análisis de estados financieros se hace principalmente por medio de ratios. Ya en los años sesenta del siglo pasado 
aparecieron las primeras investigaciones que demostraban la utilidad de los ratios para predecir la evolución de la empresa (Beaver, 1968). Como resultado de esta aportación, urgieron un sinnúmero de trabajos aplicando cada vez modelos estadísticos más complejos considerando los ratios financieros como variables independientes predictivas de la solvencia o bien de los rendimientos económicos. Altman, (1968) aplicó el modelo de análisis discriminante múltiple a diferencia de Beaver, quien utilizó el modelo de análisis discriminante simple. La aportación del modelo Z-Score de Altman, (1968), es ampliamente utilizado actualmente, por ser sencillo pues se requiere para su aplicación sólo información contable. Otras aportaciones han incluido además de los ratios financieros, variables como las de mercado, económicas, tamaño de la empresa, flujo de caja, estructura financiera y rendimiento de la empresa (Ohlson, 1980; Aharoni, et.al, 1980, Zmijewski, 1984; Frydman, Altman y Kao, 1985; Gombola, et. al, 1987; Altman y Heine, 1995) por considerar que en una empresa emergen aspectos multidimensionales, estos trabajos persiguen identificar qué variables independientes explican con mayor detalle el éxito o fracaso de una compañía.

En 1996, García, M. y Jiménez, S. conceptualizan el análisis financiero como un proceso de juicio crítico que consiste en: 1) realizar una acumulación e integración de información financiera, 2) formular hipótesis de trabajo para su contrastación, y 3) presentar una opinión que sea de utilidad para que la toma de decisiones sea eficiente y se mejore la asignación de recursos económicos. Asimismo, para Ehrhardt y Brigham (2009) el análisis de los estados financieros consiste en realizar un comparativo con el desempeño de la compañía con el promedio de otras compañías del mismo sector; también en evaluar las tendencias de la situación financiera a través del tiempo, ayudando a los ejecutivos a identificar las debilidades de las empresas.

Asimismo, el análisis de estados financieros evalúa la composición y relaciones de la información contable como la situación financiera, estado de resultados y de flujos de efectivo de un negocio. Esta actividad es una de las principales funciones de la gerencia, cuya finalidad es generar valor a la empresa para sus propietarios. De igual forma es de interés para los acreedores bancarios quienes aplican la medición de ciertos ratios para conocer el nivel de solvencia de los solicitantes de crédito; los accionistas e inversionistas institucionales se interesan en conocer las predicciones de ingresos y retornos futuros para compraventa de acciones y/o bonos, (Platikanova, P. 2005).

Oliveras, E. y Moya, S. (2005), consideran que el desarrollo del análisis financiero consiste en tres fases: en primer lugar, se deben seleccionar los indicadores que han demostrado mayor fortaleza en estudios empíricos, como los ratios relacionales con variables como éxito empresarial, solvencia, rentabilidad, tamaño de la empresa, entre otros. En segundo lugar, se analiza el comportamiento histórico de los últimos 4 ó 5 años de los resultados de los ratios seleccionados $\mathrm{y}$, por último, consideran que es conveniente compararla con el promedio del sector o bien con el principal competidor de éxito con características similares de giro económico y tamaño. Afirman que los ratios financieros sectoriales son la referencia para diseñar objetivos deseables desde la gerencia.

Aunque el análisis financiero comparativo 


\section{Cuadro 1. Ratios financieros utilizados en trabajos empíricos.}

\begin{tabular}{|c|c|c|}
\hline Autor (Año) & Ratios Financieros & Descripción \\
\hline Beaver (1968) & Flujo de caja / Deudas & Predictor de solvencia. \\
\hline \multirow[t]{6}{*}{ Altman (1968) } & Fondo de maniobra/ Activo & \multirow[t]{6}{*}{ Predictor de solvencia. } \\
\hline & Beneficio neto retenido / Activo & \\
\hline & BAII / Activo & \\
\hline & Valor de mercado de la empresa/ Deudas & \\
\hline & Ventas / Activos & \\
\hline & BAII / Pago total de intereses & \\
\hline \multirow[t]{3}{*}{ Zmijewski (1984) } & Ingresos netos / Activo & \multirow{3}{*}{$\begin{array}{l}\text { Predictor de solvencia o } \\
\text { modelo de quiebra. }\end{array}$} \\
\hline & Deuda / Activo & \\
\hline & Activo circulante / Pasivo circulante & \\
\hline \multirow{4}{*}{$\begin{array}{l}\text { Frydman, Altman y } \\
\text { Kao ( 1985) }\end{array}$} & Flujo de caja / Deuda & \multirow[t]{4}{*}{ Predictor de solvencia. } \\
\hline & Beneficio retenido/ Activo & \\
\hline & Deuda / Activo & \\
\hline & Tesorería / Activo & \\
\hline \multirow[t]{4}{*}{ Altman y Heine (1995) } & Capital neto de trabajo/ Activo total & \multirow{4}{*}{$\begin{array}{l}\text { Análisis de crédito para } \\
\text { mercados emergentes }\end{array}$} \\
\hline & Utilidades retenidas/ Activo total & \\
\hline & BAII / Activo total & \\
\hline & Capital Contable / Pasivo Total & \\
\hline \multirow[t]{3}{*}{ Mora, A. (1995) } & Beneficio + Amortizaciones / Pasivo total & \multirow{3}{*}{$\begin{array}{l}\text { Indicadores fuertes para la } \\
\text { predicción del fracaso } \\
\text { empresarial }\end{array}$} \\
\hline & Rentabilidad & \\
\hline & Liquidez & \\
\hline \multirow[t]{10}{*}{ Mosqueda, R. (2004) } & $\begin{array}{l}\text { Rentabilidad del ejercicio }=(\text { Resultado neto del ejercicio } \\
\text { / Activos totales }) * 100\end{array}$ & \multirow{10}{*}{$\begin{array}{l}\text { Ratios para aumentar la } \\
\text { consistencia del cálculo de } \\
\text { un Ratio de Valoración } \\
\text { Ponderado (RPV) para } \\
\text { empresas mexicanas. }\end{array}$} \\
\hline & $\mathrm{CC} / \mathrm{AT}$ & \\
\hline & Ventas totales / AT & \\
\hline & $\begin{array}{l}\text { Tasa de crecimiento operativo }=(\text { NOPLAT actual }- \\
\text { NOPLAT año anterior)/ NOPLAT año anterior }\end{array}$ & \\
\hline & Costo de ventas / Inventarios & \\
\hline & $\mathrm{AC}$ - Inventarios / PC & \\
\hline & $\mathrm{PT} / \mathrm{CC}$ & \\
\hline & $\mathrm{CC} /($ ventas totales / 360) & \\
\hline & Utilidad del ejercicio / CC & \\
\hline & $\begin{array}{l}\text { Variación porcentual UPA / Variación porcentual } \\
\text { Utilidad antes de intereses e impuestos }\end{array}$ & \\
\hline Mosqueda, R. (2005) & Presión financiera $=$ Gasto financiero $/$ Ventas totales & \multirow{2}{*}{$\begin{array}{l}\text { Especificación del RPV. } \\
\text { Predicción de insolvencia } \\
\text { empresarial. }\end{array}$} \\
\hline $\begin{array}{l}\text { Taboada, L.; Moura, } \\
\text { W. y Douglas, R. } \\
(2009)\end{array}$ & $\begin{array}{l}\text { Resultado de la actividad / Activo total } \\
\text { Resultado de la explotación / Gastos financieros } \\
\text { Resultado de las actividades ordinarias / Ventas }\end{array}$ & \\
\hline \multirow[t]{9}{*}{$\begin{array}{l}\text { Labatut, G.; Pozuelo, } \\
\text { J. y Veres, E. (2009) }\end{array}$} & $\begin{array}{l}\text { Resultado antes de intereses e impuestos /Gastos } \\
\text { Financieros }\end{array}$ & \multirow{9}{*}{$\begin{array}{l}\text { Ratios más eficaces y } \\
\text { rápidos para detectar } \\
\text { alarmas de fracaso } \\
\text { empresarial. }\end{array}$} \\
\hline & Resultado del ejercicio/ Ventas & \\
\hline & Cash Flow recursos generados / Ventas & \\
\hline & Resultado antes de impuestos / Ventas & \\
\hline & Resultado antes de intereses e impuestos/ Ventas & \\
\hline & Resultado de actividades ordinarias/ Ventas & \\
\hline & Valor añadido / ventas & \\
\hline & Pasivo fijo / Ventas & \\
\hline & Activo circulante / Pasivo circulante & \\
\hline \multirow{3}{*}{$\begin{array}{l}\text { Mongrut,S.;Alberti, F.; } \\
\text { Fuenzalida, D. y } \\
\text { Akamine, M. (2011). }\end{array}$} & Retorno sobre activos $=$ BAIT $(1-t) /$ Activo neto & \multirow{3}{*}{$\begin{array}{l}\text { Predicción de insolvencia } \\
\text { empresarial. }\end{array}$} \\
\hline & promedio & \\
\hline & Coeficiente de solvencia $=$ Total Pasivo $/$ patrimonio & \\
\hline
\end{tabular}


a partir de ratios financieros sectoriales es relevante para la toma de decisiones financieras, en México, la propuesta de bases de datos con esta información es relativamente escasa respecto a otros países. La empresa de consultoría, ACUS, Consultores, S.C, desde 1994 publica una base de datos de razones financieras sectoriales, actualizada hasta 2007, apareciendo en la página de internet www. acus.com.mx. Este tipo de información es relevante cuando se requiere tener una visión global de los sectores, permitiendo con ello analizar comparadamente a cada empresa con su sector económico.

Por otro lado, una de las principales empresas especializadas que oferta información financiera de empresas privadas es la Bolsa Mexicana de Valores (BMV), que publica una base de datos titulada: Información financiera trimestral histórica (5 años). Ésta contiene la información financiera trimestral (Balance y Estado de Resultados) de las emisoras listadas en BMV de los años 2004-2008, excepto municipios, estados y fideicomisos.

El Instituto Nacional de Estadística y Geografía (INEGI), publica bases de datos relativos a censos económicos cada cinco años, mediante la aplicación de instrumentos diferenciados con base en métodos estadísticos. Estas bases de datos están publicadas en la página de internet del INEGI, www.inegi.org.mx, así como también está en venta un CD interactivo con esta información. Éste contiene información sobre las características de los establecimientos productores de bienes, comercializadores de mercancías y prestadores de servicios a nivel nacional, con un gran nivel de detalle geográfico y sectorial. Las variables que presenta son: datos de identificación, ubicación geográfica, número de unidades económicas, forma de organización, inicio de actividades y total de días trabajados, personal ocupado, remuneraciones, gastos, ingresos, valor de la producción, existencias, activos fijos, créditos y cuentas bancarias.

Adicional a los censos se ofrece información sobre la identificación y ubicación de todos los establecimientos activos en territorio nacional mexicano de acuerdo a los datos recabados durante la actualización de unidades económicas, 2010, mediante el Directorio Estadístico Nacional de Unidades Económicas (DENUE, 03\2011), éste cuenta con 4'374,600 de empresas registradas a nivel nacional.

Otra publicación gubernamental es el Sistema de Información Empresarial Mexicano (SIEM), cuya página de internet es www.siem.org.mx, donde se informa mediante un directorio de empresas, a los clientes potenciales, proveedores, herramientas gerenciales y la publicación de los programas de apoyo oficiales para el desarrollo de los negocios en el país.

El Instituto Mexicano de Ejecutivos de Finanzas, A.C. (IMEF) publica libros, boletines de prensa, boletines técnicos, ponencias, revistas entre otros.

La empresa privada Economatica oferta la suscripción de un software que contiene información financiera, económica y de mercado de las empresas listadas en las Bolsas de México, Estados Unidos de América, Brasil, Argentina, Chile, Perú, Colombia y Venezuela. Se actualiza al cierre de cada sesión.

También, la revista Expansión publica anualmente el ranking de las 500 empresas más importantes de México. Se presenta en 
Cuadro 2. Confiabilidad del instrumento de investigación.

\begin{tabular}{llll}
\hline Objetivos de la investigación & Variables & Reactivos del cuestionario \\
\hline Generalidades & $\begin{array}{l}\text { Sectores económicos, Tamaño y } \\
\text { régimen fiscal. }\end{array}$ & P.1 a la P.3 \\
\hline $\begin{array}{l}\text { Ratios financieros } \\
\text { ideales }\end{array}$ & sectoriales & $\begin{array}{l}\text { Ratios financieros que se aplican en } \\
\text { las empresas de Culiacán, Sinaloa, } \\
\text { México. }\end{array}$ & P. 15 a la P.39 \\
\hline Fórmulas de los ratios & $\begin{array}{l}\text { Ratios financieros y sus fórmulas } \\
\text { que se aplican en las empresas de } \\
\text { Culiacán, Sinaloa, México. }\end{array}$ & P. 15 a la P. 39 \\
\hline $\begin{array}{l}\text { Pertinencia de un anuario } \\
\text { ratios financieros sectoriales }\end{array}$ & $\begin{array}{l}\text { Tipos de bases de datos } \\
\text { Análisis financiero }\end{array}$ & $\begin{array}{l}\text { Opinión sobre la pertinencia de una } \\
\text { base de datos de ratios financieros }\end{array}$ & P.13, P.14, P.42, P.43 \\
\hline
\end{tabular}

su página de internet www.cnnexpansion. com, de manera interactiva, es decir, tiene la opción de cambiar la lista con base en las variables siguientes: clasificación por ventas netas (mdp), empleos, activo total (mdp), ubicación, patrimonio neto (mdp), sector, utilidad de operación (mdp), utilidad neta (mdp) y notas.

De lo expuesto, se desprende que no existe en México una base de datos que contenga ratios sectoriales, excepto para las empresas cotizadas y para las mayores empresas. No hay una publicación anual de ratios financieros sectoriales en México, que contenga un conjunto de ratios ideales y que se actualice hasta el último año fiscal, lo que permite enunciar la hipótesis sobre la pertinencia de un anuario de ratios financieros sectoriales en México para realizar análisis comparativo empresarial. Por lo consiguiente, los objetivos de este trabajo son tres: 1) describir los ratios financieros ideales para el análisis por sectores económicos de empresas mexicanas, 2) detallar las fórmulas de cada ratio financiero, 3) diseñar un instrumento para medir la pertinencia de la base de datos de ratios financieros sectoriales como herramienta para el análisis financiero en las empresas ubicadas en Culiacán, Sinaloa,
México. Este artículo de investigación muestra resultados temporales, quedando pendiente la aplicación del cuestionario diseñado.

\section{MATERIALES Y MÉTODOS}

Para seleccionar los ratios financieros con mayor poder predictivo, se consideran los materiales de trabajos empíricos desarrollados por un número importante de investigadores a nivel internacional, que han aplicado los ratios como determinantes para predecir la insolvencia empresarial, fracasos o éxitos en las organizaciones (ver cuadro 1). La metodología es descriptiva, es decir, se seleccionan los ratios que se ha demostrado que tienen más poder predictivo en estudios previos, para la composición de la base de datos de ratios financieros sectoriales de las empresas mexicanas.

Para diseñar el cuestionario que permita conocer la pertinencia de la publicación anual de una base de datos de ratios financieros sectoriales en Culiacán, Sinaloa, México, se consideraron las siguientes variables de estudio: 1) la utilización de bases de datos de información financiera, 2) aplicación de ratios o razones financieras para el 
Cuadro 2. Tamaño de la muestra estratificada.

\begin{tabular}{|c|c|c|c|c|}
\hline Sector & $\begin{array}{l}\text { Unidades } \\
\text { económicas } \\
\text { Sinaloa }\end{array}$ & Factor & $\begin{array}{l}\text { Tamaño } \\
\text { muestral } \\
\text { estratificado } \\
\text { según } \\
\text { formula } \\
\end{array}$ & $\begin{array}{l}\text { Tamaño } \\
\text { muestral } \\
\text { estratificado } \\
\text { final }\end{array}$ \\
\hline Construcción & 667 & 0.00057834 & 0.38 & 2 \\
\hline Industria manufacturera & 8171 & 0.00057834 & 4.73 & 5 \\
\hline Comercio al por mayor & 3171 & 0.00057834 & 1.83 & 2 \\
\hline Comercio al por menor & 33593 & 0.00057834 & 19.42 & 20 \\
\hline $\begin{array}{l}\text { Servicios inmobiliario y de alquiler de } \\
\text { bienes muebles e intangibles }\end{array}$ & 1456 & 0.00057834 & 0.84 & 2 \\
\hline $\begin{array}{l}\text { Servicios de esparcimiento culturales y } \\
\text { deportivos y otros servicios recreativos }\end{array}$ & 996 & 0.00057834 & 0.57 & 2 \\
\hline $\begin{array}{l}\text { Servicios de alojamiento temporal y de } \\
\text { preparación de alimentos y bebidas }\end{array}$ & 8110 & 0.00057834 & 4.69 & 5 \\
\hline $\begin{array}{l}\text { Otros servicios excepto actividades } \\
\text { gubernamentales }\end{array}$ & 12959 & 0.00057834 & 7.49 & 8 \\
\hline Total & 69,123 & & 39.97 & 46 \\
\hline
\end{tabular}

Fuente: Censos económicos 2009. INEGI.

análisis y 3) la opinión sobre la pertinencia de la publicación de un anuario sobre ratios financieros sectoriales en México y si lo utilizarían para realizar análisis financiero comparativo. El instrumento de investigación está compuesto por 43 reactivos, de los cuales 37 son preguntas cerradas y 6 preguntas abiertas. El diseño del cuestionario se realiza con en el software Dyane (Diseño y análisis de encuestas) versión 4, pues éste facilita la recopilación y análisis de la información. El instrumento se somete a revisión por pares de expertos para su validez. La confiabilidad se demuestra mediante la consistencia de la relación entre objetivos, variables y reactivos según el cuadro 2.

El tamaño de la muestra para aplicar el instrumento en cuestión, se determina con base en el número de unidades económicas en Sinaloa, publicadas por el INEGI, en el censo económico 2009. Los sectores para calcular el tamaño de la muestra estratificado son los que aparecen en el cuadro 3, éstos coinciden en su mayoría con los sectores que aparecerán en la publicación propuesta del anuario de ratios financieros sectoriales (ver cuadro 4).
La determinación del tamaño de la muestra según el muestreo probabilístico estratificado, se calcula con la siguiente fórmula estadística:

$\mathrm{N}=$ El tamaño de la población 69123 empresas en Sinaloa.

$\mathrm{Y}=$ Valor promedio de la variable $=1.0000$

$\mathrm{Se}=$ Error estándar propuesto $=0.15$

$\mathrm{Se} 2=$ Varianza de la población $=0.0225$

$\mathrm{S} 2=$ Probabilidad de ocurrencia $=0.90$

n' $=$ tamaño de la muestra $\sin$ ajustar $=\mathrm{S} 2 /$ $\mathrm{Se} 2=40$

$\mathrm{n}=$ tamaño de la muestra $=(40 /((1+(40 / 69$ 123))) $=40$

Factor de estratificación $=\mathrm{n} / \mathrm{N}=40 / 69123$ $=0.00057834$

El tamaño de la muestra estratificada según la formula estadística da como resultado que 40 encuestas son suficientes para generalizar las opiniones de los empresarios en Culiacán, Sinaloa. Sin embargo, por acuerdo de los encargados de esta investigación se aplicarán 46 cuestionarios en total, sumatoria que resultó del redondeo y de la estrategia de encuestar a 2 empresarios como mínimo por cada sector económico (ver cuadro 2). 
La selección de los encuestados será al azar con base en la información de empresas del Directorio Estadístico Nacional de Unidades Económicas (DENUE, 03\2011).

\section{Presentación de los resultados}

Se realizó la selección de los ratios con base en el enfoque deductivo. Es decir, se consideró lo planteado por las propuestas de estudios empíricos (ver cuadro 1) y también por la experiencia de los autores de este trabajo. Los ratios seleccionados son los que han demostrado su potencial como predictores de solvencia, análisis de crédito y fracaso empresarial. Se clasifican en: liquidez, endeudamiento, gestión de activos, ciclo económico, rendimiento y capacidad de autofinanciación. Los ratios financieros que se seleccionaron para el anuario son los siguientes:

\section{Liquidez}

La liquidez es la capacidad que tiene la empresa para atender sus compromisos de corto plazo. Los ratios que se han seleccionado han demostrado ser predictores de insolvencia desde Beaver en 1968 hasta Mongrut, Fuenzalida y Akamine (2011). Cada vez los investigadores proponen un menor número de ratios predictores de éxito en una empresa. Otros estudios proponen ratios predictores más eficientes y rápidos para detectar el fracaso empresarial (Labatut, Pozuelo y Veres, 2009).

$\begin{aligned} \text { Circulante }= & \text { Activo circulante } \\ & \text { Pasivo circulante }\end{aligned}$

Prueba rápida $=$ Activo circulante Inventarios

\section{Pasivo circulante}

Capital neto de trabajo $=$ Activo circulante Pasivo circulante
Pasivo circulante

Disponible $=$ Efectivo e inversiones a corto plazo

Pasivo circulante

Coeficiente de solvencia $=$ Pasivo total

Capital contable

\section{Endeudamiento}

Los ratios de endeudamiento miden el nivel de deuda comparada con la aportación de los socios. Los tipos de deuda se miden con los ratios de deuda a corto y largo plazo. El ratio de cobertura de gastos financieros es aplicado para conocer cuántas veces alcanza cubrir los intereses con el rendimiento de la actividad principal del negocio. Para Mosqueda, R. (2005) el ratio de presión financiera es determinante en la especificación del RPV (ver cuadro 1).

Endeudamiento $=$ Capital contable Pasivo total

Deuda a corto plazo $=$ Pasivo a corto plazo Pasivo total

Deuda a largo plazo $=$ Pasivo a largo plazo Pasivo total

Cobertura de gastos financieros $=$ BAII Gastos financieros

Presión financiera $=$ Gastos financieros Ventas totales

\section{Ratios de gestión de activos}

Estos miden la eficiencia con que se están utilizando los activos para generar ventas. La rotación del activo total, activo fijo neto $\mathrm{y}$ activo circulante, mide las veces en que éstos se recuperan mediante las ventas; 
estos ratios son considerados predictores de solvencia, su monitoreo evita el fracaso en las empresas y generan valor, (Altman, 1968; Amat, O., Leiva, LI. y Graells, J., 2002); Mosqueda, R., 2004).

Rotación del activo $=$ Ventas netas $\quad$ x 100 Activos totales

Rotación del activo fijo $=$ Ventas netas $\quad \mathrm{x}$ 100

\section{Activo fijo neto}

Rotación del activo circulante $=$ Ventas netas $\mathrm{x} 100$

circulante

Composición de activos:

Activo circulante

Activo total

Activo fijo neto

Activo total

\section{Ratios del ciclo económico}

El ciclo económico es la suma de los días en que tarda el inventario en salir para su venta y los días de recuperación de las cuentas por cobrar. A esta sumatoria se le restan los días que la empresa tarda en pagarle a sus proveedores. Este ciclo debe ser el más corto posible. Es importante mencionar que cada sector tiene promedios de ciclos económicos diferentes debido a su actividad.

Días de inventarios $=$ Inventarios $* 365$ Costo de ventas

Días de cuentas por cobrar $=\mathrm{CXC} * 365$ Ventas netas

Días de proveedores $=$ Proveedores $* 365$
Costo de ventas

Ciclo financiero $=$

Días de inventarios + Días de cuentas por cobrar

- Días de proveedores.

\section{Ratios de rendimiento}

El rendimiento en una empresa de lucro es su razón de ser. Los mejores predictores de éxito son los que en su numerador tienen las cuentas de resultados o beneficios, todos los citados en el cuadro 1 proponen algún ratio considerando los beneficios retenidos, antes de intereses e impuestos o beneficio antes de impuestos, en el numerador. Labatut, Pozuelo y Veres (2009), proponen como ratio más rápido para evitar fracasos el retorno líquido.

Rentabilidad operativa $=$ BAII

Activo total

Rentabilidad financiera $=$ Beneficio neto Capital contable

Retorno sobre ventas $=$ Beneficio neto Ventas

Retorno líquido $=$ Flujo de caja Ventas

Ratios de capacidad de autofinanciación Beaver (1968) y Frydman, Altman y Kao (1985) consideran en el numerador el cash flow tradicional relacionándolo con las deudas totales como predictores de solvencia. En cambio, Amat, Leiva y Graells (2002), lo proponen en el numerador para conocer el grado de autofinanciación sobre los activos y las ventas.

Capacidad de autofinanciación sobre activo $=$ 
Beneficio neto + Depreciación $y$ amortización

Activo total

Capacidad de autofinanciación sobre ventas $=$

Beneficio neto + Depreciación $y$ amortización

Ventas

Cabe mencionar que el anuario de ratios financieros sectoriales contendrá la clasificación de los ratios anteriormente expuestos. Se realizaron algunos cálculos para determinar la factibilidad técnica de las fórmulas, éstos fueron con base en la información financiera dictaminada del periodo 2006-2010, de las empresas listadas enlaBMV delabase de datos deEconomatica. Con este software también se seleccionaron los sectores económicos, que se incluirán, siendo éstos los que cuenten con más de 2 empresas como mínimo, las cuales sumaron 86 empresas privadas no financieras (ver cuadro 3). No se considerarán las empresas financieras y las de seguros dado que tienen normativas contables diferentes y formas de funcionar que las hacen no asimilables al resto de sectores incluidos en el estudio. Las 86 empresas son relativamente pocas en proporción al total de unidades económicas en el país, sin embargo, sus ingresos totales en el periodo 2003-2010 (Economatica, 2011), representan el $21.43 \%$ del PIB nacional (INEGI). Por tanto, las empresas seleccionadas representan una parte significativa de la actividad económica de México. Se calculó en el SPSS mediante regresión lineal simple el coeficiente de correlación corregido por 0.997 , lo que indica una alta relación positiva entre los ingresos netos y el PIB en el periodo mencionado. Justificándose con ello, la importancia económica y financiera de las empresas listadas en la BMV, y que pueden marcar la tendencia del sector empresarial nacional por lo que se justifica la realización de un anuario de ratios financieros basado en estas empresas.

Asimismo, según información del censo económico 2009 del INEGI, son tres sectores económicos los de mayor importancia en cuanto al número de unidades económicas. En primer lugar, el sector comercio con el $49.9 \%$ del total, integrado por el $46.7 \%$ del subsector comercio al por menor y el $3.2 \%$ de comercio al por mayor. En segundo lugar, está el sector de servicios privados no financieros con el 36.2\% de participación. Y por último, en tercera posición, se encuentra la industria manufacturera con el $11.7 \%$ de participación en las unidades económicas. De éste, el 33\% corresponde al subsector de la industria alimentaria, el $14.7 \%$ a la fabricación de productos metálicos y el 7.6\% al subsector, de la fabricación de prendas de vestir. Se observa en el cuadro 3, que del tamaño total de la muestra estratificada por sectores para la base de datos propuesta en este trabajo, el $64.34 \%$ están comprendidos en los tres sectores de mayor importancia señalados por el INEGI. El 10.85\% corresponde al comercio al por menor, el $3.1 \%$ al comercio de mayoreo; el $21.71 \%$ a las empresas dedicadas a otorgar servicios privados no financieros y el $31.78 \%$ a la industria manufacturera.

Con lo expuesto, se cumple con los dos primeros objetivos de este estudio; el tercer objetivo se logró con la presentación del siguiente instrumento de investigación el cual será aplicado a 46 empresarios de Culiacán, Sinaloa: 


\section{Cuestionario}

El presente cuestionario está dirigido a las personas encargadas de generar información para el análisis financiero en la toma de decisiones de la empresa, quienes pueden ser por ejemplo, el dueño, socios, gerente, administrador, contador $\mathrm{u}$ otros afines. El objetivo es conocer la pertinencia de la publicación anual de una base de datos de ratios financieros sectoriales con base en la información financiera de las empresas que cotizan en la Bolsa Mexicana de Valores; que resulte de interés para aplicarla en sus empresas como una referencia para la comparación. Los encargados de esta investigación se comprometen que las respuestas serán presentadas de manera general por lo que se respetará la confidencialidad. En ningún momento se difundirán datos de la identidad de las personas que apoyaron con este trabajo. Agradecemos de antemano su distinguida colaboración.

\section{Cuadro 3. Selección de los sectores económicos para el anuario de ratios financieros sectoriales en México.}

\begin{tabular}{|ll}
\hline Sectores Económicos & Núm. Empresas \\
\hline Minería & 5 \\
\hline Construcción y emprendimientos inmobiliarios & 10 \\
\hline Construcción pesada & 4 \\
\hline Industria alimentaria & 10 \\
\hline Industria de las bebidas & 5 \\
\hline Industria química & 3 \\
\hline Industria del papel & 2 \\
\hline Fabricación de productos a base de minerales no metálicos & 7 \\
\hline Fabricación de productos metálicos & 4 \\
\hline Fabricación de maquinaria y equipo & 2 \\
\hline Comercio al por mayor & 4 \\
\hline Comercio al por menor (Tiendas de mercancías diversas ) & 9 \\
\hline Tiendas de artículos de salud y cuidados personales & 2 \\
\hline Servicios relacionados con el transporte aéreo & 5 \\
\hline Radio, Televisión y otras telecomunicaciones & 10 \\
\hline Servicios de alojamiento temporal & 4 \\
\hline Total & $\mathbf{8 6}$ \\
\hline
\end{tabular}

Fuente: Economatica, 2011.

Instrucciones: Se solicita atentamente dar respuesta a las preguntas acorde a la realidad de su empresa.

P. 1. Actividad económica principal de la empresa

P. 2. Número de trabajadores

P. 3. Régimen fiscal 
¿Cuales son las bases de datos de información empresarial que utiliza?

\begin{tabular}{|c|c|c|c|c|c|c|}
\hline Tipos de bases de datos: & Nunca & $\begin{array}{l}1 \text { vez al } \\
\text { año }\end{array}$ & $\begin{array}{l}\text { Semestr } \\
\text { al }\end{array}$ & $\begin{array}{l}\text { Cuatri } \\
\text { mestral }\end{array}$ & $\begin{array}{l}\text { Trimest } \\
\text { ral }\end{array}$ & $\begin{array}{l}\text { Mensua } \\
1\end{array}$ \\
\hline $\begin{array}{l}\text { P.4. Información financiera de empresas } \\
\text { listadas en la Bolsa Mexicana de Valores. }\end{array}$ & $\square 1$ & $\square 2$ & $\square 3$ & $\square 4$ & $\square 5$ & $\square 6$ \\
\hline $\begin{array}{l}\text { P. 5. Información estadística publicada por } \\
\text { el Instituto Nacional de Estadística y } \\
\text { Geografía (INEGI) }\end{array}$ & $\square 1$ & $\square 2$ & $\square 3$ & $\square 4$ & $\square 5$ & $\square 6$ \\
\hline $\begin{array}{l}\text { P. 6. Sistema de Información Empresarial } \\
\text { (SIEM) }\end{array}$ & $\square 1$ & $\square 2$ & $\square 3$ & $\square 4$ & $\square 5$ & $\square 6$ \\
\hline $\begin{array}{l}\text { P. 7. Publicaciones realizadas por } \\
\text { consultores especialistas en análisis de } \\
\text { empresas. }\end{array}$ & $\square 1$ & $\square 2$ & $\square 3$ & $\square 4$ & $\square 5$ & $\square 6$ \\
\hline P. 8. Software para análisis financiero. & $\square 1$ & $\square 2$ & $\square 3$ & $\square 4$ & $\square 5$ & $\square 6$ \\
\hline $\begin{array}{l}\text { P. 9. Publicaciones del Instituto Mexicano } \\
\text { de Ejecutivos de Finanzas (IMEF) }\end{array}$ & $\square 1$ & $\square 2$ & $\square 3$ & $\square 4$ & $\square 5$ & $\square 6$ \\
\hline P. 10. Revista 'Expansión' & $\square 1$ & $\square 2$ & $\square 3$ & $\square 4$ & $\square 5$ & $\square 6$ \\
\hline P. 11. Economatica & $\square 1$ & $\square 2$ & $\square 3$ & $\square 4$ & $\square 5$ & $\square 6$ \\
\hline
\end{tabular}

P. 12. Especificar otras bases de datos que utilicen en su empresa y no hayan sido enunciadas en la pregunta anterior.

P. 13. ¿Realizan análisis de empresas aplicando ratios o razones financieras?

1. Si

2. No

P. 14. En caso de que la respuesta anterior fuera negativa, ¿Cuáles son las causas por las que no aplican este tipo de análisis?

\begin{tabular}{|c|c|c|}
\hline Ratios financieros & $\mathbf{S i}$ & No \\
\hline $\begin{array}{r}\text { P. 15. Circulante }=\underbrace{\text { Activo circulante }}_{\text {Pasivo circulante }} \\
\text { circula }\end{array}$ & $\square 1$ & $\square 2$ \\
\hline P. 16. Prueba rápida $=\frac{\text { Activo circulante }- \text { Inventarios }}{\text { Pasivo circulante }}$ & $\square 1$ & $\square 2$ \\
\hline $\begin{array}{l}\text { P. 17. Capital neto de trabajo }= \\
\text { Activo circulante }- \text { Pasivo circulante } \\
\text { Pasivo circulante }\end{array}$ & $\square 1$ & $\square 2$ \\
\hline P. 18. Disponible $=\frac{\text { Efectivo e inversiones a corto plazo }}{\text { Pasivo circulante }}$ & $\square 1$ & $\square 2$ \\
\hline $\begin{array}{l}\text { P. 19. Coeficiente de solvencia }=\text { Pasivo total } \\
\text { Capital contable }\end{array}$ & $\square 1$ & $\square 2$ \\
\hline P. 20. Endeudamiento $=\frac{\text { Capital contable }}{\text { Pasivo total }}$ & $\square 1$ & $\square 2$ \\
\hline P. 21. Deuda a corto plazo $=\frac{\text { Pasivo a corto plazo }}{\text { Pasivo total }}$ & $\square 1$ & $\square 2$ \\
\hline
\end{tabular}




\begin{tabular}{|c|c|c|}
\hline P. 22. Deuda a largo plazo $=\frac{\text { Pasivo a largo plazo }}{\text { Pasivo total }}$ & $\square 1$ & $\square 2$ \\
\hline $\begin{array}{l}\text { P. 23. Cobertura de gastos financieros }=\underline{B A I I} \\
\text { Gastos financieros }\end{array}$ & $\square 1$ & $\square 2$ \\
\hline P. 24. Presión Financiera $=\frac{\text { Gastos financieros }}{\text { Ventas totales }}$ & $\square 1$ & $\square 2$ \\
\hline P. 25. Rotación del activo $=\frac{\text { Ventas netas } \times 100}{\text { Activos totales }}$ & $\square 1$ & $\square 2$ \\
\hline P. 26. Rotación del activo fijo $=\frac{\text { Ventas netas } \times \quad 100}{\text { Activo fijo neto }}$ & $\square 1$ & $\square 2$ \\
\hline $\begin{array}{l}\text { P. 27. Rotación del activo circulante }= \\
\frac{\text { Ventas netas } \times 100}{\text { Activo circulante }}\end{array}$ & $\square 1$ & $\square 2$ \\
\hline $\begin{array}{l}\text { P. 28. Composición de activos } \frac{\text { Activo circulante }}{\text { Activo total }}+ \\
\frac{\text { Activo Fijo Neto }}{\text { Activo total }}=100 \%\end{array}$ & $\square 1$ & $\square 2$ \\
\hline P. 29. Días de inventarios $=\underline{\text { Costo de ventas }}$ & $\square 1$ & $\square 2$ \\
\hline P. 30. Días de cuentas por cobrar $=\underset{\text { Ventas netas }}{\mathrm{CXC} * 365}$ & $\square 1$ & $\square 2$ \\
\hline P. 31. Días de proveedores $=\underline{\text { Proveedores } * 365}$ & $\square 1$ & $\square 2$ \\
\hline $\begin{array}{l}\text { P. 32. Ciclo financiero = Días de inventarios }+ \text { Días de cuentas por cobrar }- \\
\text { Días de proveedores }\end{array}$ & $\square 1$ & $\square 2$ \\
\hline $\begin{array}{r}\text { P. 33. Rentabilidad operativa }=\underline{\text { BAII }} \\
\text { Activo total }\end{array}$ & $\square 1$ & $\square 2$ \\
\hline P. 34. Rentabilidad financiera Beneficio neto & $\square 1$ & $\square 2$ \\
\hline
\end{tabular}

\begin{tabular}{|c|c|c|}
\hline P. 35. Retorno sobre ventas $=\frac{\text { Beneficio neto }}{\text { Ventas }}$ & $\square 1$ & $\square 2$ \\
\hline P. 36. Retorno líquido $=\frac{\text { Flujo de caja recursos generados }}{\text { Ventas }}$ & $\square 1$ & $\square 2$ \\
\hline $\begin{array}{l}\text { P. 37. Capacidad de autofinanciación sobre activo }= \\
\frac{\text { Beneficio neto }+ \text { depreciación y amortización }}{\text { Activo total }}\end{array}$ & $\square 1$ & $\square 2$ \\
\hline $\begin{array}{l}\text { P. 38. Capacidad de autofinanciación sobre ventas }= \\
\frac{\text { Beneficio neto }+ \text { depreciación y amortización }}{\text { Ventas }}\end{array}$ & $\square 1$ & $\square 2$ \\
\hline
\end{tabular}

P. 39. En caso de que utilicen otros ratios financieros que no se mencionaran en la pregunta anterior, favor de especificarlos: 
P. 40. En caso de aplicar ratios financieros, ¿Cuáles referencias consideran para comparar los ratios financieros del negocio?

1. Contra la historia misma del negocio

2. Promedio del sector económico

3. Ratios definidos por la gerencia

4. Otros

\begin{tabular}{|l|l|l|}
\hline P. 41. ¿Reciben asesorías externas para diagnósticos y análisis de empresas? & Si & No \\
\hline $\begin{array}{l}\text { P. 42. ¿Considera pertinente la publicación de un anuario sobre ratios } \\
\text { financieros sectoriales con base en las empresas que se encuentran listadas en }\end{array}$ & $\square 1$ & $\square 2$ \\
la Bolsa Mexicana de Valores? & $\square 2$ \\
$\begin{array}{l}\text { P. 43. En caso de que se publicara un anuario sobre ratios financieros } \\
\text { sectoriales en México, ¿lo aplicaría para el análisis financiero de la empresa } \\
\text { donde labora? }\end{array}$ & $\square 1$ & $\square 2$ \\
\hline
\end{tabular}

\section{DISCUSIÓN}

Gallizo y Salvador (2000), realizaron un estudio sectorial para medir las fuerzas de equilibro de seis ratios significativos para análisis de empresas, éstos fueron recabados de la publicación de la Central de Balances del Banco de España. Con base en el modelo de ajuste parcial calcularon el grado de similitud de los coeficientes de ajuste. Los resultados obtenidos identificaron un alto grado de similitud entre los coeficientes de ajuste y demostraron que la velocidad de ajuste no está relacionada con el tamaño y tipo de las firmas. El sector energético mostró en sus ratios una alta sensibilidad ante los acontecimientos externos. Gallizo y Salvador, demostraron con sus resultados que las empresas no necesariamente tienen porque objetivar los ratios, pues éstos se ajustan mayormente a las fuerzas externas del mercado y al comportamiento del propio sector.

Aunque el estudio anterior pareciera limitar la importancia del análisis financiero sectorial, se destaca que esta herramienta es aplicada para el análisis de estados financieros como parte de un diagnóstico global de cualquier entidad económica, el cual consiste en conocer la eficiencia del desempeño empresarial como una de las actividades primordiales de los directivos. Este análisis radica en que el analista selecciona ratios con base en los estados contables que le permiten formarse una idea de la visión en conjunto de la empresa, los resultados se consideran como parte integral de un diagnóstico global de una empresa al que se incluye además la perspectiva estratégica y operativa, Amat, O.(2005). En coincidencia, López Lubián, F. (2005), propone monitorear el desempeño empresarial con base en métricas que contengan los generadores de valor económico desde el modelo del Cuadro de Mando Integral (Balanced Scorecard). Para ello, considera indicadores de valor extrínseco e intrínseco, con la finalidad de que exista una convergencia entre los objetivos de corto y largo plazo. Las actuaciones de gestión directiva mediante las metas de ratios con base en los promedios del sector industrial es parte importante de su desempeño en las empresas. 
De igual manera el trabajo realizado por Samaniego, Trujillo y Martín (2007) demostraron con una muestra de 105 empresas cotizadas en el mercado continuo español al 31 de diciembre de 2004, las diferencias en puntuación crediticia que proporcionan la Z Score y el modelo de Merton. Así, obtuvieron que en un gran número de empresas existía cierto grado de asociación lineal positiva entre las calificaciones crediticias proporcionadas por el modelo contable y el basado en el mercado. Solo encontraron discrepancias en el sector tecnológico afirmando que quizá sea porque el valor de mercado está más influido por las expectativas que por el análisis histórico. Lo que permite, identificar la importancia de la información contable clasificada en ratios financieros sectoriales promedio para análisis y monitoreo del desempeño empresarial.

\section{CONCLUSIONES}

Del trabajo efectuado se desprende la importancia de las bases de datos con ratios financieros sectoriales para mejorar el análisis financiero de las empresas. También se ha identificado la laguna que existe en este terreno en México. Por ello, se propone la publicación anual de una base de datos con ratios financieros sectoriales del conjunto de empresas que cotizan en el mercado de valores mexicano por ser relevante para los analistas de estados financieros según el estudio teórico fundamentado.

La clasificación ideal por ratios es la siguiente: liquidez, endeudamiento, gestión de activos, ciclo económico, rendimiento y capacidad de autofinanciación. Con los cálculos de estas razones financieras es factible realizar un diagnóstico global en un negocio. En este estudio se han presentado las fórmulas respectivas con la finalidad de investigar con los empresarios de Culiacán, Sinaloa, México, cuáles son las que aplican para realizar análisis financiero y si están interesados en monitorear sus ratios con el promedio de la industria que se publicará en el anuario. Es preciso aplicar los cuestionarios para investigar la pertinencia de esta propuesta en el campo de acción. Esto permitirá identificar cuál es la pertinencia de una publicación de esta naturaleza, la cual no existe en el país.

\section{LITERATURA CITADA}

Aharony, J.; Jones, C.P.; y Swary, I. 1980. An Analysis of Risk and Return Characteristics of Corporate Bankruptcy Using Capital Market Data. The Journal of Finance. Vol. 35, No. 4, pp. 1001-1016.

Altman, E. 1968. Financial Ratios, Discriminant Analysis and the Prediction of Corporate Bankruptcy. The Journal of Finance. Vol. 23, No. 4, pp. 589-609.

Altman, E.I. y Heine, M.L. 1995. Emerging Market Corporate Bonds _ A Scoring System. Research Emergin Markets. Salomon, Brothers, INC. Revisada el 20 de octubre de 2011. http://pages.stern.nyu.edu/ ealtman/ Emerg_Mkt_Corporate_Bonds.pdf

Amat, O. 2005. Claves del análisis de empresas. Contabilidad y Dirección. Vol.2. pp. 13-51. Análisis de empresas. Editorial Deusto. Barcelona.

Amat, O.; Leiva, LI. y Graells, J. 2002. Ratios sectoriales. Ediciones Gestión 2000. Barcelona. España.

Beaver, W. 1968. Market Prices, Financial Ratios, and the Prediction of Failure. 
Journal of Accounting Research. Vol. 6, No. 2, pp. 179-192.

Ehrhardt, M. y Brigham, E. 2009. Finanzas Corporativas. Editorial CENGAGE Learning. México.

Frydman, H.; Altman, E.I. y Y Kao, D. 1985. Introducing Recursive Partitioning for Financial Classification: The Case of Financial Distress. The Journal of Finance. Vol. 40, No. 1, pp. 269-291.

Gallizo, J.L. y Salvador, M. 2000. Medida del proceso de ajuste de los ratios financieros. Un análisis en sectores industriales. Revista Española de Financiación y Contabilidad, Vol. XXIX, Núm. 103, Enero-Abril, pp. $37-56$.

García, M. y Jiménez, S. 1996. Una reflexión crítica sobre el concepto y ámbito de análisis financiero y los objetivos de la investigación en materia de análisis de la información financiera. Revista Española de Financiación y Contabilidad. Vol. XXVI, Núm. 87, pp. 403-427.

Gombola, M.J.; Haskins, M.E.; Ketz, J.E. y Williams, D.D. 1987. Cash Flow in Bankruptcy Prediction. Financial Management. Vol. 16, No. 4, pp. 5565.

Labatut, G.;Pozuelo, J. y Veres, E.2009. Modelización temporal de los ratios contables en la detección del fracaso empresarial de la PyME Española. Revista Española de Financiación y Contabilidad. Vol. XXXVIII, N ${ }^{\circ}$ 143, pp. 423-447.

López Lubián, F. 2005. Indicadores de valor en el Balanced Scorecard. Revista de Empresa. Núm. 14, pp. 94-106. Retrieved from EBSCOhost. http:// search.ebscohost.com/login.aspx?di rect $=$ true $\& d b=z b h \& A N=26304159$ \&la

Mongrut,S.; Alberti, F.; Fuenzalida, D. y Akamine, M. 2011. Determinantes de la Insolvencia Empresarial en el Perú. Academia Latinoamericana de Administración, Vol. 47, pp. 126139.

Mora, A. 1995. Utilidad de los Modelos de Predicción de la crisis empresarial. Revista Española de Financiación y Contabilidad. Vol. XXIV, no ${ }^{\circ}$ 83, pp. 281-300.

Mosqueda R. 2004. Propuesta de un ratio ponderado de valoración empresarial. IV Workshop de Investigación Empírica en Contabilidad Financiera, Universidad de Sevilla. Revisada el 22 de octubre de 2011. http://www. aloj.us.es/arquero/docs/15.pdf

Mosqueda R. 2005. Valoración de los indicadores del Fracaso en las empresas mexicanas. Modelo ponderado de valoración del riesgo. Investigación Financiera Empresarial. Revisada el 22 de octubre de 2011. http:// www.imef.org.mx/fundacion/ premionacional/2005/empresarial/ mencion/valoracion.pdf

Ohlson, J.A. 1980. Financial Ratios and the Probabilistic Prediction of Bankruptcy. Journal of Accounting Research. Vol. 18, No.1, pp. 109131.

Oliveras, E. y Moya, S. 2005. La utilización de los datos sectoriales para complementar el análisis de los estados financieros. Contabilidad y Dirección. Vol.2. pp. 53-69. Tema: Análisis de empresas. Editorial Deusto. Barcelona. España.

Platikanova, P. 2005. El análisis económico- 
financiero: Estado del arte. Contabilidad y Dirección. Vol.2. pp. 13-51. Tema: Análisis de empresas. Editorial Deusto. Barcelona. España.

Samaniego, R.; Trujillo, A. y Martin, J.L. 2007. Un análisis de los modelos contables y de mercado en la evaluación del riesgo de crédito: aplicación al mercado bursátil español. Revista Europea de Dirección y Economía de la Empresa, Vol. 16, nº 2, pp.93-110.

Taboada, L.; Moura, W. y Douglas, R. La información contable en la predicción de insolvencia: un estudio inferencial aplicado a empresas industriales cotizadas. Revista Contemporânea de Contabilidade. Vol. 6, $\mathrm{N}^{\mathrm{o}}$ 12, pp. 151-170.

Zmijewski, M.E. 1984. Methodological Issue Related to the Estimation of Financial Distress Prediction Models. Journal of Accounting Research. Vol. 2, Studies on Current Econometric Issues in Accounting Research, pp. 59-82.

Páginas de internet: www.acus.com.mx, www.bmv.com.mx, www.inegi.org. mx, www.siem.org.mx, www.imef. org.mx, www.cnnexpansion.com

\section{Software}

Economatica de México

Diseño y Análisis de Encuestas (DyANE, Versión 4) SPSS 17

Deyanira Bernal Domínguez, Doctora en Ciencias Sociales por la UAS. Profesora e investigadora de Tiempo Completo de la Facultad de Contaduría y Administración de la UAS. Correo electrónico: deyanirabernaldominguez@gmail. com, Celular: 6677757293

Oriol Amat Salas, Dr. En Ciencias Económicas por la UAB International. Profesor e investigador de la Universidad Pompeu Fabra, Catedrático de Economía Financiera y Contabilidad, Barcelona, España. 